\title{
Indicação química de possível ocorrência de Florencita-(Y) em Bom Sucesso (Minas Gerais, Brasil)
}

\author{
Chemical indication of Florencite-(Y) occurrence at Bom Sucesso \\ (Minas Gerais, Brazil)
}

Alexandre de Oliveira Chaves ${ }^{1 *}$

RESUMO: A florencita- $(\mathrm{Y})$, sinônimo do mineral desacreditado koivinita-(Y), representa uma espécie até então não reconhecida pela Associação Mineralógica Internacional. Com base em análises em microssonda eletrônica, há uma indicação química da possível ocorrência deste mineral no município de Bom Sucesso (MG, Brasil). Esta provável espécie mineral foi encontrada associada a churchita- $(\mathrm{Y})$ em granitoide leucocrático potássico, peraluminoso, foliado, de 2,7 Ga, do núcleo arqueano da porçăo meridional do Craton São Francisco. A fórmula obtida para esta presumível florencita de ítrio é $\left(\mathrm{Y}_{0.402} \mathrm{Nd}_{0.213} \mathrm{La}_{0.171} \mathrm{Ca}_{0.086} \mathrm{Dy}_{0.044} \mathrm{Gd}_{0.032} \mathrm{Ce}_{0.029} \mathrm{Sm}_{0.029} \mathrm{Pr}_{0}\right.$. $\left.{ }_{018} \mathrm{Fe}_{0.017} \mathrm{~Pb}_{0.008} \mathrm{Th}_{0.001} \mathrm{U}_{0.001}\right)_{\Sigma 1.051} \mathrm{Al}_{3.002}\left(\mathrm{P}_{0.959} \mathrm{Si}_{0.039} \mathrm{O}_{4}\right)_{2}(\mathrm{OH})_{6.073}$. Com relação à churchita- $(\mathrm{Y})$ a ela associada, sua fórmula é $\left(\mathrm{Y}_{0.475} \mathrm{La}_{0.184} \mathrm{Nd}_{0}\right.$. $\left.{ }_{123} \mathrm{Ca}_{0.055} \mathrm{Gd}_{0.043} \mathrm{Dy}_{0.029} \mathrm{Sm}_{0.028} \mathrm{Pr}_{0.023} \mathrm{Fe}_{0.017} \mathrm{Ce}_{0.016} \mathrm{Al}_{0.007} \mathrm{~Pb}_{0.007} \mathrm{U}_{0.002} \mathrm{Th}_{0.002}\right)$ ${ }_{\Sigma 1011}\left(\mathrm{P}_{0984} \mathrm{Si}_{0021}\right) \mathrm{O}_{4} \cdot 2 \mathrm{H}_{2} \mathrm{O}$. Uma vez que o granitoide peraluminoso de Bom Sucesso não mostra sinais de argilizaçấo intempérica de feldspatos, seus principais minerais constituintes, sugere-se que a suposta florencita- $(\mathrm{Y})$ nele encontrada seja de origem primária, ou seja, formada durante a cristalizaçáo (ou recristalizaçáo) da rocha.

PALAVRAS-CHAVE: florencita- $(\mathrm{Y})$, koivinita- $(\mathrm{Y})$, Bom Sucesso, Minas Gerais.

\section{INTRODUÇÃO}

Segundo Bayliss et al. (2010), espécies minerais do supergrupo da alunita $\left[\mathrm{KAl}_{3}\left(\mathrm{SO}_{4}\right)_{2}(\mathrm{OH})_{6}\right]$ têm a fórmula química geral $\mathrm{DG}_{3}\left(\mathrm{TX}_{4}\right)_{2} \mathrm{X}_{6}$, em que $\mathrm{D}$ é um cátion tetravalente, trivalente, divalente, monovalente ou uma vacância parcial; G é um cátion trivalente e por vezes cátions bivalentes; $T$ é um cátion hexavalente, pentavalente $\mathrm{e}$, às vezes $\mathrm{Si}^{4+}$; $\mathrm{eX} / \mathrm{X}^{\prime}$ é $\mathrm{O},(\mathrm{OH})$, às vezes $\mathrm{F}$ e, eventualmente, $\mathrm{H}_{2} \mathrm{O}$, como mostrado na Tab. 1. Estas espécies foram identificadas e descritas ao longo dos últimos dois séculos.
ABSTRACT: Florencite- $(Y)$, synonymous with the discredited koivinite- $(Y)$, represents a mineral species not recognized by International Mineralogical Association. Based on electron microprobe analyses, there is a chemical indication of the apparent occurrence of such mineral at Bom Sucesso (Minas Gerais State, Brazil). This supposed mineral species has been found in association with churchite-(Y) in $2.7 \mathrm{Ga}$ potassic peraluminous foliated granitoid from the Archean core of the southern portion of the São Francisco Craton. The formula obtained for this probable yttrium florencite is $\left(Y_{0.402} \mathrm{Nd}_{0.213} \mathrm{La} a_{0.171} \mathrm{Ca} a_{0.086} \mathrm{Dy} \mathrm{y}_{0.044} \mathrm{Gd}_{0.032} \mathrm{Ce}_{0.029} \mathrm{Sm}_{0.029} \mathrm{Pr}_{0.018} \mathrm{Fe}_{0.017} \mathrm{P}\right.$ $\left.b_{0.008} \mathrm{Th}_{0.001} U_{0.001}\right)_{51.051} \mathrm{Al}_{3.002}\left(P_{0.959} \mathrm{Si}_{0.039} \mathrm{O}_{4}\right)_{2}(\mathrm{OH})_{6.073}{ }^{\circ} \quad$ Regarding churchite-(Y), its formula is $\left(Y_{0.475} L a_{0.184} N d_{0.123} C a_{0.055} G d_{0.043} D y_{0.0}\right.$ $\left.{ }_{29} \mathrm{Sm}_{0.028} \mathrm{Pr}_{0.023} \mathrm{Fe}_{0.017} \mathrm{Ce} e_{0.016} \mathrm{Al} \mathrm{l}_{0.007} \mathrm{~Pb} b_{0.007} U_{0.002} \mathrm{Th}_{0.002}\right)_{51.011}\left(P_{0.984} \mathrm{Si}_{0.021}\right)$ $\mathrm{O}_{4} .2 \mathrm{H}_{2} \mathrm{O}$. Given that the Bom Sucesso peraluminous granitoid shows no signs of argillic weathering of feldspars, it is suggested a primary origin for the apparent florencite- $(Y)$, i.e., it has been formed during crystallization (or recrystallization) of the rock.

KEYWORDS: florencite-(Y), koivinite-(Y), Bom Sucesso, Minas Gerais State.

A florencita (nome em homenagem ao mineralogista brasileiro William Florence, 1864-1942, que fez o exame químico preliminar da primeira florencita) é um fostato de elementos terras-raras leves deste supergrupo, no qual o sítio D foi até agora descrito como predominantemente ocupado por Ce, La, Nd ou Sm, com Al no sítio G (Repina et al. 2011). As diferentes espécies de florencita conhecidas cristalizam-se no sistema trigonal (classe escalenoédrica hexagonal), fazem parte do grupo da plumbogummita $\left[\mathrm{PbAl}_{3}\left(\mathrm{PO}_{3.5}(\mathrm{OH})_{0.5}\right)_{2}(\mathrm{OH})_{6}\right]$ (Mills et al. 2009) e seus termos extremos estáo listados na Tab. 2.

${ }^{1}$ Centro de Pesquisas Manoel Teixeira da Costa, Departamento de Geologia, Instituto de Geociências, Universidade Federal de Minas Gerais - UFMG, Belo Horizonte (MG), Brasil. E-mail: alex2010@ufmg.br

*Autor correspondente

Manuscrito ID: 30195. Recebido em: 06/11/2014. Aprovado em: 14/11/2014. 
Tabela 1. Elementos químicos e vacâncias (símbolo do quadrado) que podem ocupar os sítios cristalográficos D, G, T e X das espécies minerais do supergrupo da alunita, DG3(TX4)2X'6. Em cada sítio, eles estão listados em ordem decrescente de valência (Bayliss et al. 2010).

\begin{tabular}{|c|c|}
\hline $\mathrm{D}$ & $\begin{array}{l}\text { Th, Ce, La, Nd, Sm, Bi, Ca, Sr, Ba, Pb, Hg, Na, K, Rb, Ag, Tl, } \\
\mathrm{NH}_{4}, \mathrm{H}_{3} \mathrm{O},\end{array}$ \\
\hline G & $\mathrm{Sn}^{4+} ; \mathrm{Al}, \mathrm{Fe}^{3+}, \mathrm{V}^{3+}, \mathrm{Cr}^{3+}, \mathrm{Ga} ; \mathrm{Cu}^{2+}, \mathrm{Zn}, \mathrm{Mg}$ \\
\hline $\mathrm{T}$ & $\mathrm{S}^{6+}, \mathrm{Cr}^{6+} ; \mathrm{P}, \mathrm{As}, \mathrm{Sb} ; \mathrm{Si}$ \\
\hline $\mathrm{X}$ & $\mathrm{O} ;(\mathrm{OH}), \mathrm{F} ;\left(\mathrm{H}_{2} \mathrm{O}\right)$ \\
\hline
\end{tabular}

Tabela 2. Espécies de florencita conhecidas e as fórmulas químicas teóricas de seus termos extremos.

\begin{tabular}{c|c}
\hline Florencita-(Ce) & $\mathrm{CeAl}_{3}\left(\mathrm{PO}_{4}\right)_{2}(\mathrm{OH})_{6}$ \\
\hline Florencita-(La) & $\mathrm{LaAl}_{3}\left(\mathrm{PO}_{4}\right)_{2}(\mathrm{OH})_{6}$ \\
\hline Florencita-(Sm) & $\mathrm{SmAl}_{3}\left(\mathrm{PO}_{4}\right)_{2}(\mathrm{OH})_{6}$ \\
\hline Florencita-(Nd) & $\mathrm{NdAl}_{3}\left(\mathrm{PO}_{4}\right)_{2}(\mathrm{OH})_{6}$ \\
\hline
\end{tabular}

A florencita- $(\mathrm{Y})$, sinônimo do mineral koivinita-(Y) relatado na obra de Clark (1993) e que foi desacreditado pela comunidade científica (Burke 2006), representa uma espécie, até então, não reconhecida pela Associação Mineralógica Internacional (IMA - International Mineralogical Association). O objetivo do presente trabalho é apresentar a indicação de uma composição química de uma provável florencita de ítrio que ocorre associada a churchita-(Y) em granitoide leucocrático na região de Bom Sucesso (MG, Brasil) e discutir sua gênese.

O referido granitoide faz parte de um grupo de rochas similares potássicas peraluminosas foliadas, com idade entre 2,75 - 2,70 Ga (Romano et al. 2013), geradas por anatexia de gnaisses migmatíticos do setor meridional do Craton São Francisco. O arcabouço geológico em que o granitoide se insere está apresentado na Fig. 1, que mostra o núcleo cratônico arqueano

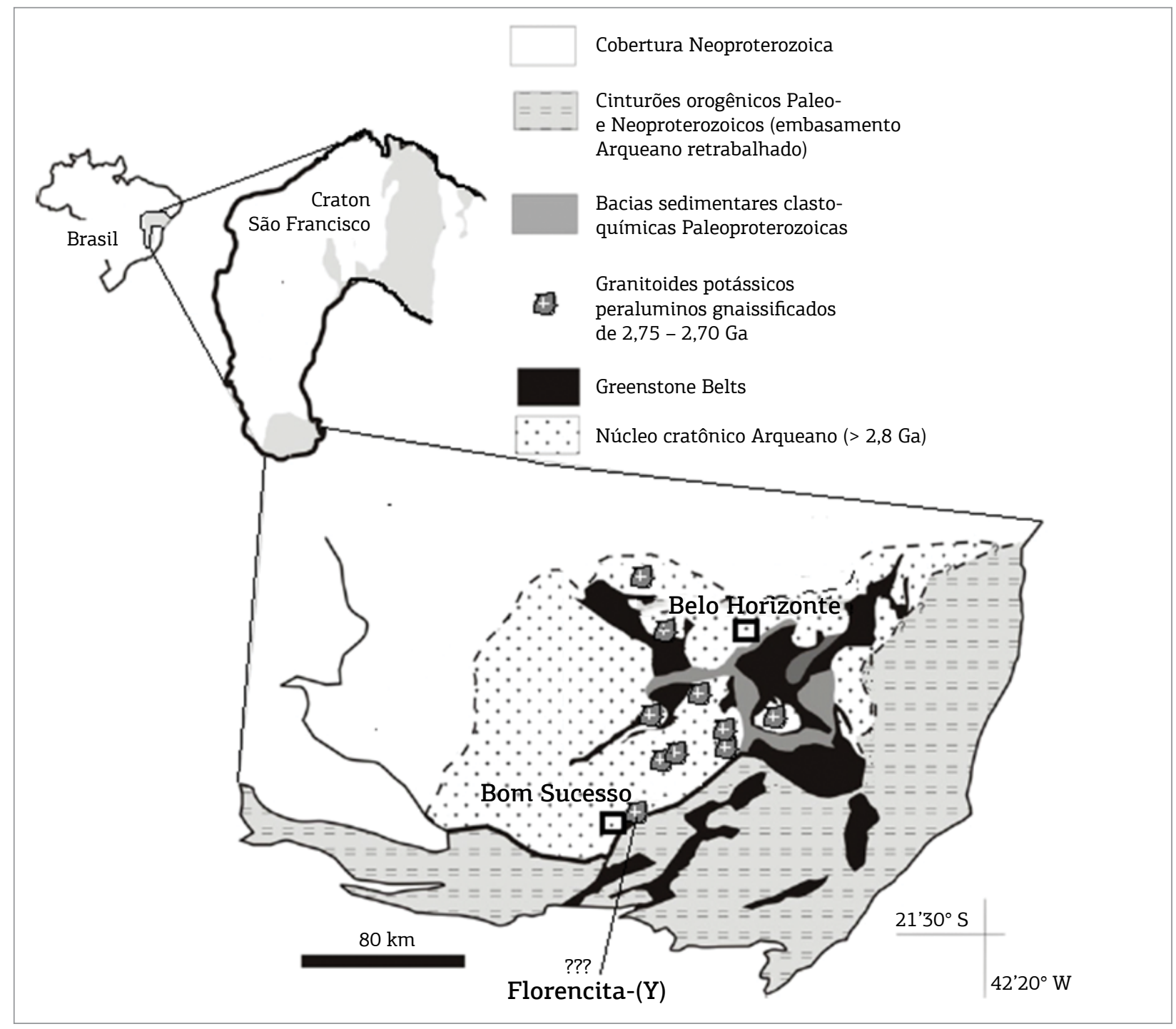

Figura 1. Localização do granitoide de Bom Sucesso (MG), no qual foi encontrada a suposta florencita-(Y), em seu contexto geológico regional do setor sul do Craton São Francisco. Fonte: modificado de Romano et al. 2013. 
com idades superiores a 2,8 Ga, greenstone belts arqueanos e paleoproterozóicos, bacias sedimentares clasto-químicas paleoproterozóicas, o embasamento arqueano retrabalhado ao longo de cinturôes orogênicos paleo- e neoproterozóicos e a cobertura sedimentar neoproterozóica sobre o Craton São Francisco.

\section{MATERIAIS E MÉTODOS}

No Centro de Pesquisas Manoel Teixeira da Costa do Instituto de Geociências da Universidade Federal de Minas Gerais (UFMG) foi feita a lâmina delgada polida da amostra do granitoide foliado, que foi utilizada nos estudos micropetrográficos em microscópio polarizador. Após sua pulverização em moinho de panela, o granitoide foi enviado ao Laboratório SGS - Geosol para a obtenção da composição de seus elementos maiores por meio da técnica ICP-OES (Plasma indutivamente acoplado a espectrômetro de emissáo óptica). A perda ao fogo ocorreu por diferença de peso após aquecimento a $1.000^{\circ} \mathrm{C}$.

Utilizando a mesma lâmina delgada polida, em uma microssonda JEOL - modelo JXA-8900 - instalada no Laboratório de Microanálises da UFMG, foram obtidas a imagem de elétrons-retroespalhados e as composiçôes químicas dos cristais de churchita e da presumível florencita analisados. Os parâmetros para análises pontuais quantitativas utilizados encontram-se na Tab. 3 .

\section{RESULTADOS}

A partir dos estudos micropetrográficos, pode ser observado que o granitoide leucocrático de granulação média de Bom Sucesso, no qual encontra-se a suposta florencita-(Y), é predominantemente composto por microclínio e quartzo (com extinção ondulante), aparecendo plagioclásio albítico e biotita em menor volume. Ele mostra textura granolepidoblástica e feições de recristalização. Raras ilmenita e florencita de ítrio (?) correspondem aos acessórios. Em termos modais, é um álcali-feldspato granito que se encontra metamorficamente foliado, ou seja, é um gnaisse granítico rico em K-feldspato. Ressalte-se que não há sinais de argilização dos minerais no granitoide estudado. Sua composição química, em termos de elementos maiores, encontra-se na Tab. 4. A simples

Tabela 3. Parâmetros empregados nas microanálises dos cristais estudados.

\begin{tabular}{|c|c|c|c|c|c|c|c|}
\hline \multicolumn{8}{|c|}{ Método = WDS (espectrometria por dispersão de comprimento de onda) } \\
\hline \multicolumn{8}{|c|}{ Voltagem de aceleração da coluna eletrônica $=25 \mathrm{Kv}$} \\
\hline \multicolumn{8}{|c|}{ Intensidade da corrente $=50 \mathrm{nA}$} \\
\hline \multicolumn{8}{|c|}{ Diâmetro do feixe de elétrons = 2 micrômetros } \\
\hline \multirow{2}{*}{ Elemento } & \multirow{2}{*}{ Raio-X } & \multirow{2}{*}{ Cristal } & \multicolumn{2}{|c|}{ Tempo de contagem } & \multicolumn{2}{|c|}{ Ofsets background } & \multirow{2}{*}{ Padrão analítico } \\
\hline & & & Pico & Background & Bg L & Bg U & \\
\hline Y & La & TAP & 20,0 & 10,0 (seg) & 1,12 & 0,84 & YAG - Granada de Y e Al \\
\hline Dy & $\mathrm{Lb}$ & LIF & 20,0 & 10,0 (seg) & 0,84 & 0,00 & $\mathrm{DyPO}_{4}$ \\
\hline $\mathrm{P}$ & $\mathrm{Ka}$ & PETJ & 10,0 & 5,0 (seg) & 0,70 & 0,70 & Monazita \\
\hline $\mathrm{Si}$ & $\mathrm{Ka}$ & TAP & 10,0 & 5,0 (seg) & 1,38 & 0,84 & $\mathrm{ThSiO}_{4}$ thorita sintética \\
\hline Gd & $\mathrm{Lb}$ & LIF & 20,0 & 10,0 (seg) & 0,84 & 0,98 & $\mathrm{GdPO}_{4}$ \\
\hline $\mathrm{Pb}$ & $\mathrm{Mb}$ & PETJ & 20,0 & 10,0 (seg) & 1,64 & 0,64 & Crocoita \\
\hline $\mathrm{Fe}$ & $\mathrm{Ka}$ & LIF & 10,0 & 5,0 (seg) & 0,70 & 0,84 & Hematita \\
\hline Th & $\mathrm{Ma}$ & PETJ & 20,0 & 10,0 (seg) & 1,95 & 0,84 & $\mathrm{ThSiO}_{4}$ thorita sintética \\
\hline $\mathrm{Sm}$ & $\mathrm{Lb}$ & LIF & 20,0 & 10,0 (seg) & 0,55 & 0,53 & REE2 \\
\hline $\mathrm{U}$ & $\mathrm{Mb}$ & PETJ & 20,0 & 10,0 (seg) & 0,00 & 2,00 & $\mathrm{UO}_{2}$ sintético \\
\hline $\mathrm{Al}$ & $\mathrm{Ka}$ & TAP & 20,0 & 10,0 (seg) & 1,12 & 0,84 & YAG - Granada de Y e Al \\
\hline $\mathrm{Ca}$ & $\mathrm{Ka}$ & PETJ & 10,0 & 5,0 (seg) & 0,84 & 0,70 & $\mathrm{Ca}_{2} \mathrm{P}_{2} \mathrm{O}_{7}$ apatita sintética \\
\hline $\mathrm{Nd}$ & $\mathrm{Lb}$ & LIF & 20,0 & 10,0 (seg) & 0,84 & 0,84 & $\mathrm{NdPO}_{4}$ \\
\hline $\mathrm{La}$ & $\mathrm{La}$ & PETJ & 10,0 & 5,0 (seg) & 0,84 & 0,84 & Monazita \\
\hline $\operatorname{Pr}$ & $\mathrm{Lb}$ & LIF & 20,0 & 10,0 (seg) & 0,84 & 0,66 & $\mathrm{PrPO}_{4}$ \\
\hline $\mathrm{Ce}$ & $\mathrm{La}$ & LIF & 10,0 & 5,0 (seg) & 0,84 & 1,12 & Monazita \\
\hline
\end{tabular}

Tabela 4. Composição química do granitoide de Bom Sucesso, expressa em porcentagem dos óxidos de seus elementos maiores (percentagem em peso).

\begin{tabular}{c|c|c|c|c|c|c|c|c|c|c|c}
\hline $\mathrm{SiO}_{2}$ & $\mathrm{TiO}_{2}$ & $\mathrm{Al}_{2} \mathbf{O}_{3}$ & $\mathbf{F e O}$ & $\mathbf{M g O}$ & $\mathbf{M n O}$ & $\mathbf{C a O}$ & $\mathbf{K}_{2} \mathbf{O}$ & $\mathbf{N a}_{2} \mathbf{O}$ & $\mathbf{P}_{2} \mathbf{O}_{5}$ & $\mathbf{P F}$ & Total \\
\hline 75,03 & 0,34 & 13,03 & 2,04 & 0,25 & 0,01 & 0,33 & 5,31 & 1,88 & 0,10 & 2,04 & 100,36 \\
\hline
\end{tabular}

PF: perda ao fogo. Fe total apresentado como FeO. 
observação dos elevados valores de $\mathrm{K}_{2} \mathrm{O}$ e $\mathrm{Al}_{2} \mathrm{O}_{3}$ da tabela confirma o caráter potássico e peraluminoso (índice de alumina-saturação A/CNK = 1,4) da rocha.

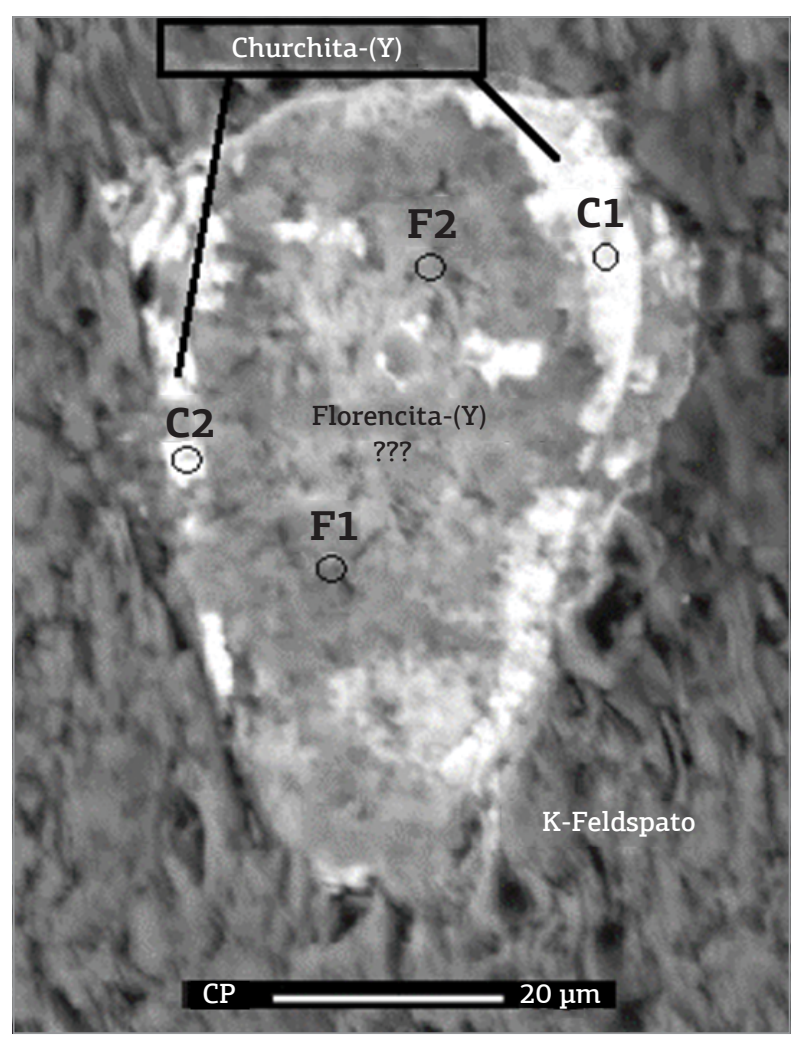

Figura 2. Imagem de elétrons retro-espalhados da suposta florencita-(Y) bordejada pela churchita-(Y), no interior de microclínio do granitoide potássico peraluminoso de Bom Sucesso (MG). A textura rugosa em toda a imagem indica que o polimento, infelizmente, não foi muito bem sucedido sobre esta diminuta área da seção. F1, F2, C1 e C2 são os pontos analisados.
Com relação à hipotética florencita-(Y), foco deste estudo, a imagem de elétrons retro-espalhados obtida na microssonda (Fig. 2) revela que a mesma encontra-se subédrica e bordejada por outro mineral (cor branca na Fig. 2) mais rico em átomos de elementos de elevado peso atômico do que ela, ambos no interior de microclínio. Este outro mineral corresponde aparentemente à churchita-(Y) e o resultado das microanálises que mostra a composição de ambos está apresentado na Tab. 5.

Foram feitas duas análises na pretensa florencita-(Y), correspondentes aos pontos F1 e F2 da Fig. 2 e duas na churchita-(Y), correspondentes aos pontos $\mathrm{C} 1$ e $\mathrm{C} 2$ da mesma figura. $\mathrm{O}$ total de cátions por unidade de fórmula, com base em 14 ânions oxigênio para a florencita e seis ânions oxigênio para a churchita, estão também apresentados na Tab. 5 e foram calculados a partir da média de duas análises em cada mineral. Os conteúdos de $\mathrm{H}_{2} \mathrm{O}$ não analisados pela microssonda foram considerados com base no conteúdo teórico de água, igual a dois por unidade de fórmula na churchita, e de grupos $\mathrm{OH}$, igual a seis por unidade de fórmula na florencita.

A partir dos valores de cada cátion por unidade de fórmula da florencita da Tab. 5, nota-se ser ítrio (Y) o cátion dominante, o que eventualmente tornaria esta florencita numa espécie nova. A fórmula desta suposta florencita-(Y), é:

$$
\begin{aligned}
& \left(\mathrm{Y}_{0.402} \mathrm{Nd}_{0.213} \mathrm{La}_{0.171} \mathrm{Ca}_{0.086} \mathrm{Dy}_{0.044} \mathrm{Gd}_{0.032} \mathrm{Ce}_{0.029} \mathrm{Sm}_{0.029}\right. \\
& \left.\mathrm{Pr}_{0.018} \mathrm{Fe}_{0.017} \mathrm{~Pb}_{0.008} \mathrm{Th}_{0.001} \mathrm{U}_{0.001}\right)_{\Sigma 1.051} \mathrm{Al}_{3.002} \\
& \left(\mathrm{P}_{0.959} \mathrm{Si}_{0.039} \mathrm{O}_{4}\right)_{2}(\mathrm{OH})_{6.073}
\end{aligned}
$$

Com relação à churchita-(Y), a partir dos valores de cada cátion na Tab. 5, chega-se à fórmula:

$$
\begin{aligned}
& \left(\mathrm{Y}_{0.475} \mathrm{La}_{0.184} \mathrm{Nd}_{0.123} \mathrm{Ca}_{0.055} \mathrm{Gd}_{0.043} \mathrm{Dy}_{0.029} \mathrm{Sm}_{0.028} \mathrm{Pr}_{0.023} \mathrm{Fe}_{0.017}\right. \\
& \left.\mathrm{Ce}_{0.016} \mathrm{Al}_{0.007} \mathrm{~Pb}_{0.007} \mathrm{U}_{0.002} \mathrm{Th}_{0.002}\right)_{\Sigma 1.011}\left(\mathrm{P}_{0.984} \mathrm{Si}_{0.021}\right) \mathrm{O}_{4} .2\left(\mathrm{H}_{2} \mathrm{O}\right)
\end{aligned}
$$

\begin{tabular}{|c|c|c|c|c|c|c|c|c|c|c|c|c|c|c|c|c|c|c|}
\hline \multicolumn{19}{|c|}{ Suposta Florencita-(Y) } \\
\hline Ponto & $\mathrm{O}_{5}$ & $\mathrm{O}_{2}$ & ${ }_{2} \mathrm{O}_{3}$ & $\mathrm{Y}_{2} \mathrm{O}_{3}$ & $\mathrm{La}_{2} \mathrm{O}_{3}$ & $\mathrm{Ce}_{2} \mathrm{O}_{3}$ & $\mathrm{Pr}_{2} \mathrm{O}_{3}$ & $\mathrm{Nd}_{2} \mathrm{O}_{3}$ & $\mathrm{Sm}_{2} \mathrm{O}_{3}$ & $\mathrm{Dy}_{2} \mathrm{O}_{3}$ & $\mathrm{Gd}_{2} \mathrm{O}_{3}$ & $\mathrm{PbO}$ & $\mathrm{ThO}_{2}$ & $\mathrm{UO}_{2}$ & eO & $\mathrm{aO}$ & $\mathrm{I}_{2} \mathrm{O}$ & otal \\
\hline $\mathrm{F} 1$ & 88 & 99 & 86 & 0,57 & 93 & 07 & 76 & 778 & 06 & 69 & 31 & 27 & 06 & 04 & 24 & 80 & 1,0 & 30 \\
\hline 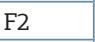 & 94 & 76 & 77 & 0,13 & & & 43 & & & & & 49 & & 04 & 25 & 14 & 1,0 & 0,35 \\
\hline ád & ,41 & 0 , & 1 & 10,35 & & & 0 , & & & & & 0,38 & & 44 & 24 & 97 & 11,0 & 0,32 \\
\hline tior & $\mathbf{P}$ & $s$ & & $\mathbf{Y}$ & & & & & & & & & & & & & $\mathrm{OH})$ & Total \\
\hline & & & & 702 & & & 0,018 & 213 & & & & 8 & & & & & 73 & \\
\hline \multicolumn{19}{|c|}{ Churchita-(Y) } \\
\hline onto & $\mathbf{P}_{2} \mathrm{O}_{5}$ & $\mathrm{SiO}_{2}$ & $\mathrm{Al}_{2} \mathrm{O}_{3}$ & $\mathrm{Y}_{2} \mathrm{O}_{3}$ & $\mathrm{La}_{2} \mathrm{O}_{3}$ & $\mathrm{Ce}_{2} \mathrm{O}_{3}$ & $\mathrm{Pr}_{2} \mathrm{O}_{3}$ & $\mathrm{Nd}_{2} \mathrm{O}_{3}$ & $\mathrm{Sm}_{2} \mathrm{O}_{3}$ & $\mathrm{Dy}_{2} \mathrm{O}_{3}$ & $\mathrm{Gd}_{2} \mathrm{O}_{3}$ & $\mathrm{PbO}$ & $\mathrm{ThO}_{2}$ & $\mathrm{UO}_{2}$ & eO & $\mathrm{aO}$ & $\mathrm{H}_{2} \mathrm{O}$ & otal \\
\hline & & & & 24,56 & & & & & & & & & & & & & 4,5 & \\
\hline$C$ & & & & & & & & & & & & & & & 21 & 0 & 4,5 & 1,42 \\
\hline & 27,91 & (5) & 5 & 24,26 & 11,95 & 1,05 & 1,53 & 8 , & 1, & 2,19 & 3,13 & 0,64 & 0,2 & 0,26 & 0,50 & 1,24 & 14,5 & 100,27 \\
\hline tions & - & $\mathbf{S i}$ & - & - & & & & & & & & $\mathrm{Pb}$ & & & Fe & $\mathrm{Ca}$ & $\mathrm{H}_{2} \mathrm{O}$ & Total \\
\hline $60 x)$ & 0,984 & 0,021 & 0,007 & 0,475 & 0,184 & 0,016 & 0,023 & 0,123 & 0,028 & 0,029 & 0,043 & 0,007 & 0,002 & 0,002 & 0,017 & 0,055 & 4,032 & 6,050 \\
\hline
\end{tabular}

Tabela 5. Composições químicas, em termos de percentagem em peso de óxidos, da suposta florencita-(Y) e da churchita-(Y) e seus respectivos cátions por unidade de fórmula, obtidos a partir da média das análises de cada mineral. 


\section{DISCUSSÃO E CONCLUSÕES}

A provável associação florencita-(Y) e churchita-(Y) apresentada na Fig. 2 sugere que o total de átomos do elemento incompatível Y praticamente se equivale ao somatório dos átomos dos também incompatíveis ETR no granitoide de Bom Sucesso, o que explicaria o fato de náo ter sido cristalizada nenhuma das já conhecidas espécies de florencita de $\mathrm{La}$, $\mathrm{Ce}, \mathrm{Nd}$ ou Sm da literatura científica. Dadas as semelhanças de raio e carga trivalente entre $Y$ e ETR, não há problemas na substituiçáo mútua entre eles, e o surgimento da florencita-(Y) neste granitoide tornou-se possível.

Emdem et al. (1997) indicaram dois mecanismos principais de compensação de cargas necessária às substituiçôes de ETR por The $\mathrm{U}$ em fosfatos de elementos terras raras (ETR) e de Y:

$$
\begin{aligned}
& \mathrm{Ca}^{2+}+\mathrm{Th}^{4+}\left(\mathrm{e} / \mathrm{ou} \mathrm{U}^{4+}\right)=2 \mathrm{ETR}^{3+} \\
& \mathrm{Si}^{4+}+\mathrm{Th}^{4+}\left(\mathrm{e} / \mathrm{ou} \mathrm{U}^{4+}\right)=\mathrm{ETR}^{3+}+\mathrm{P}^{5+}
\end{aligned}
$$

Dada a presença de Th, U e também Ca e $\mathrm{Si}$, tanto na florencita- $(\mathrm{Y})$ quanto na churchita- $(\mathrm{Y})$, certamente os mecanismos (I) e (II) se fizeram necessários para garantir o equilíbrio das cargas em ambos os minerais.

Segundo Stoffregen et al. (2000), os fosfatos aluminosos do supergrupo da alunita são, em sua maioria, formados por intemperismo de outros minerais de fosfato, como a apatita presente em rochas ígneas e metamórficas. Formam-se, ainda, por interação de águas superficiais que percolam guano, ricos em fósforo, com outras rochas ricas em alumínio. Entretanto, segundo os mesmos autores, a florencita-(ETR) tem sido encontrada em rochas não intemperizadas como carbonatitos e veios associados à intrusōes alcalinas, bem como em mica-xistos e pegmatitos graníticos peraluminosos.
Uma vez que o granitoide peraluminoso de Bom Sucesso não mostra sinais de argilização de feldspatos, seus principais minerais constituintes, sugere-se que a hipotética florencita- $(\mathrm{Y})$ nele encontrada seja de origem primária; ou seja, formada durante a cristalizaçâo (ou recristalização) desta rocha. Possivelmente, o pouco cálcio desta rocha adentrou aos cristais de plagioclásio, náo sendo suficiente para formar apatita, tendo em vista ainda o baixo teor de fósforo do granitoide. Assim sendo, o excesso de alumínio que não adentrou aos feldspatos desta rocha peraluminosa teria combinado com este pouco fósforo para formar a florencita-(Y) em ambiente hidratado. Esse ambiente primário hidratado é revelado também pela própria composição da churchita-(Y) associada à florencita- $(\mathrm{Y})$.

Fica aqui registrada a indicação química da possível ocorrência da florencita- $(\mathrm{Y}),\left(\mathrm{Y}_{0.402} \mathrm{Nd}_{0.213} \mathrm{La}_{0.171} \mathrm{Ca}_{0.086} \mathrm{Dy}_{0.044}\right.$ $\left.\mathrm{Gd}_{0.032} \mathrm{Ce}_{0.029} \mathrm{Sm}_{0.029} \mathrm{Pr}_{0.018} \mathrm{Fe}_{0.017} \mathrm{~Pb}_{0.008} \mathrm{Th}_{0.001} \mathrm{U}_{0.001}\right)_{\Sigma 1.051} \mathrm{Al}_{3.002}$ $\left(\mathrm{P}_{0.959} \mathrm{Si}_{0.039} \mathrm{O}_{4}\right)_{2}(\mathrm{OH})_{6.073}$, em Bom Sucesso (MG, Brasil), o que sugere que ao sítio $\mathrm{D}$ da Tab. 1 seja acrescentado o elemento ítrio. A determinação dos parâmetros cristalográficos e das propriedades físicas e ópticas deste presumível mineral para fins de reconhecimento pela IMA não será tarefa fácil, tendo em vista o diminuto tamanho do cristal encontrado e por ele estar entremeado à churchita-(Y), uma outra espécie mineral.

\section{AGRADECIMENTOS}

Ao CNPq, pela bolsa de produtividade em pesquisa concedida ao autor. Aos colegas do laboratório de microanálises, Luis Garcia e Márcio Flores e ao estudante Daniel Andrade Miranda.

\section{REFERÊNCIAS}

Bayliss P., Kolitsch U., Nickel E.H., Pring A. 2010. Alunite supergroup: recommended nomenclature. Mineralogical Magazine, 74(5):919-927.

Burke E.A.J. 2006. A mass discreditation of GQN minerals. The Canadian Mineralogist, 44(6):1557-1560.

Clark A.M. 1993. Hey's Mineral Index (3rd ed.). London, Chapman \& Hall.

Emdem B.V., Thornber M.R., Graham J., Lincoln F.J. 1997. The incorporation of actinides in monazite and xenotime from placer deposits in western Australia. The Canadian Mineralogist, 35:95-104.

Mills S.J., Hatert F., Nickel E.H., Ferraris G. 2009. The standardisation of mineral group hierarchies: application to recent nomenclature proposals. European Journal of Mineralogy, 21:1073-1080.
Repina S.A., Popova V.I., Churin E.I., Belogub E.V., Chiller V.V. 2011. Florencite-(Sm), $(\mathrm{Sm}, \mathrm{Nd}) \mathrm{Al}_{3}\left(\mathrm{PO}_{4}\right)_{2}(\mathrm{OH})_{6}$ - a new mineral of alunite-jarosite group from the Subpolar Urals. Geology of Ore Deposits, 53(7):564-574.

Romano R., Lana C., Alkmim F.F., Stevens G.S., Armstrong, R. 2013. Stabilization of the southern portion of the São Francisco Craton, SE Brazil, through a long-lived period of potassic magmatism. Precambrian Research, 224:143-159

Stoffregen R.E., Alpers C.N., Jambor J.L. 2000. Alunite-jarosite crystallography, thermodynamics, and geochronology. In: Alpers C.N., Jambor J.L., Nordstrom D.K. (eds.) Reviews in Mineralogy and Geochemistry, 40, St Louis, The Mineralogical Society of America, Washington,D.C. and the GeochemicalSo ciety, p. 453-479.

Arquivo digital disponível on-line no site www.sbgeo.org.br 